\title{
Effects of vacancies on high-order harmonic generation in a linear chain with band gap
}

\author{
Hossein Iravani $\odot,{ }^{1,2}$ Kenneth K. Hansen, ${ }^{1}$ and Lars Bojer Madsen $\odot^{1}$ \\ ${ }^{1}$ Department of Physics and Astronomy, Aarhus University, DK-8000 Aarhus C, Denmark \\ ${ }^{2}$ Department of Chemistry, Tarbiat Modares University, P. O. Box 14115-175, Tehran, I. R. Iran
}

(Received 5 July 2019; revised manuscript received 23 December 2019; accepted 24 January 2020; published 25 February 2020)

\begin{abstract}
High-order harmonic generation (HHG) in imperfect lattices with point defect vacancies is studied using a self-consistent time-dependent density-functional approach for a one-dimensional linear chain with a band gap. Compared with a perfect lattice with no vacancies, the HHG yield decreases by increasing the number of evenly distributed vacancies but remains almost unchanged in the case when vacancies are localized at neighboring lattice points. By introducing atomic-type point vacancies in the linear chain, it is effectively partitioned into several subsystems and the overall shape of HHG spectra of the resulting system can be modeled via the HHG of its subsystems provided each subsystem is large enough to behave like a bulk solid. For sufficiently small subsystems, the HHG spectra show detectable signatures of finite structure but maintains the overall structure of the spectrum of a bulk solids. A time-frequency profile of the emitted harmonics shows less regular sinusoidal patterns in the systems with vacancies compared with the perfect lattice. The role the vacancy-induced defectstate orbitals in the HHG process is investigated for different realizations of the linear chain and laser parameters.
\end{abstract}

DOI: 10.1103/PhysRevResearch.2.013204

\section{INTRODUCTION}

Recently, high-order harmonic generation (HHG) from solid-state systems with a band gap has received substantial attention [1-8] with potential applications for novel vacuum ultraviolet (VUV) and extreme ultraviolet (XUV) light sources and for probing ultrafast dynamics in condensedmatter systems [9]. In solid-state systems, valence and conduction band electrons are delocalized over the lattice of the crystal. The motion of electrons is therefore different than in free space and results in the energy bands being nonparabolic functions of the crystal momentum. Consequently, the HHG process and the characteristics of the signal in solids is different than that in atoms and molecules [10]. For instance, multiple plateaus appear in HHG spectra of a bulk solid due to the presence of multiple conduction bands [8].

From previous studies [10], it has emerged that some of the semiclassical concepts that were developed for strong-field physics of atoms and molecules can be applied to study the interaction of laser radiation with band-gap materials. For example, interband HHG is produced in a process resembling the atomic three-step model [11,12] and involves the following steps: (i) an electron tunnels from the valence band to the conduction band, producing a hole in the valence band; (ii) the electron and its corresponding hole propagate in the solid, driven by the external field; and (iii) they recombine and emit HHG radiation with a frequency that corresponds

Published by the American Physical Society under the terms of the Creative Commons Attribution 4.0 International license. Further distribution of this work must maintain attribution to the author(s) and the published article's title, journal citation, and DOI. to the band-gap energy at the crystal momentum at which the recollision occurs [13]. More generally, it has emerged that several aspects of the HHG process in solids can be captured by solving the time-dependent Schrödinger equation for Bloch electrons in single-active-electron models [14-21] by applying many-electron approaches such as the semiconductor Bloch equations [22-25], by time-dependent densityfunctional theory (TDDFT) [26-37], and by time-dependent Hartree-Fock theory [38].

In Ref. [30], a one-dimensional (1D) TDDFT model was introduced to establish the essentials of HHG by linearly polarized laser pulses in solids. The response of a range of system sizes, from atomic to bulk solids, was studied with this model to reveal how the signal changes from atomic to solid-state HHG [32]. In the first studies, the case of a chain of identical ions was analyzed and later real space perturbations were introduced to address new effects. In Refs. [33,36], HHG from a topological edge state was studied and it was found that the below band-gap HHG signal was enhanced substantially by the presence of a topological edge state (see also Ref. [39] for a related model study). Recently, the effects of both acceptor and donor-doping on the HHG spectra were investigated [34], and it was found that a donor-doped bandgap material, with an occupied donor state within the band gap, can enhance the overall HHG efficiency by several orders of magnitude, compared with undoped and acceptor-doped materials, where in the latter case the acceptor state within the band gap is unoccupied. Very recently, the effects of disorder were investigated by imparting random shifts of the ionic lattice positions [35], and disorder was found not to have a detrimental effect on the efficiency of the HHG process.

One of the interesting issues related to HHG in solids is how the HHG spectra and the generation process are affected by vacancies. Defects and vacancies are inevitable present 
in real solids and may also be engineered and controlled to modify properties of materials in a desired manner (see, e.g., the review [40]). Very recently it was investigated by TDDFT calculations how a single vacancy affects the HHG yield in hexagonal boron nitride [37]. The HHG spectra were found to be sensitive to whether a boron or a nitrogen atom was removed. Defect states and their occupation or not were shown to play a role, as was the case in connection with doping [34]. In this work, we go beyond the consideration of a single vacancy. In order to be able to explore a large parameter space in terms of defect concentration and arrangement, we consider a linear chain model. In this study, we will focus on the overall changes in the HHG spectra and the generation process with number and type of point defects. According to Ref. [41], there are two different types of point defects: (i) an atomic-type point defect which occupies either a point of the crystal lattice or an interstitial position and can be a substitutional impurity, an interstitial atom, or a vacancy and (ii) a molecular-type point defect which occupies several neighboring atomic or interstitial points of the crystal lattice and can be composed of an impurity molecule or a cluster of several neighboring vacancies. Introducing defects to the lattice breaks the lattice periodicity which increases the theoretical complexity $[41,42]$. We will investigate how the HHG spectrum is affected by the vacancy-type point defects either being evenly distributed, or localized at neighboring lattice points, addressing the signatures of atomic- and moleculartype defects, respectively. We also study the role of the vacancy-induced defect-state orbitals in the HHG process. Finally, we explore a second realization of our linear chain model to further investigate the physical effects associated with the vacancy-type point defects. Similarly to previous studies [30,32-36], we employ a TDDFT model to study the HHG spectra of a linear chain of ions exposed to linearly polarized laser pulses. In this approach, we go beyond the single-active electron models and take electron-electron interactions into account, at least on a mean-field level. Moreover, we can model lattices with different defect concentrations in a straightforward manner, without making additional assumptions.

This paper is organized as follows. In Sec. II, we describe the theoretical model and methods used in this work. In Sec. III, the results of our calculations are presented and discussed. Finally, we conclude in Sec. IV. Atomic units (a.u.) are used throughout unless stated otherwise.

\section{THEORETICAL MODEL AND METHODS}

In previous studies [30,32-35], a model to study HHG in a linear chain of ions was introduced. As shown in Refs. $[30,32]$, if the number of ions is large enough, then the system produces a typical band structure for a bulk solid. Moreover, the 1D system is sufficient to capture essential effects of the strong-field interaction along the linear polarization direction of the external pulse. In this model, we consider a linear chain of $N$ ions separated by the lattice constant $a$. The $i$ th ion is located at the position

$$
x_{i}=[i-(N-1) / 2] a, \quad[i=0, \ldots,(N-1)],
$$

and the ionic potential is given as

$$
v_{\text {ion }}(x)=-\sum_{i=0}^{N-1} \frac{Z_{i}}{\sqrt{\left(x-x_{i}\right)^{2}+\epsilon}},
$$

where $Z_{i}$ is the nuclear charge of the $i$ th ion and $\epsilon$ is a softening parameter which smoothens the Coulomb singularity. As in Ref. [30], we set $\epsilon=2.25, a=7$, and $Z_{i}=4$ $[i=0, \ldots,(N-1)]$ since this set of parameters was found to produce a characteristic band structure for a band-gap material. We choose a chain of $N=200$ ions as a reference system and introduce various number of vacancies at different sites of the chain by changing the charge of ions from four to zero at the considered sites. For instance, to have a vacancy on the site of the $m$ th ion in the chain, we set the charge of the $m$ th ion equal to zero $\left(Z_{m}=0\right)$ and also reduce the number of electrons by four to keep the system charge neutral. This approach for modeling vacancies is similar to the one used in Ref. [43].

All the studied systems are charge and spin neutral. Thus the number of electrons with opposite spin is $N_{\downarrow}=N_{\uparrow}=2 \mathrm{~N}$ for a system without vacancies and $N_{\downarrow}=N_{\uparrow}=2(N-M)$ for a system with $M$ vacancies. We determine the field-free electronic states for these systems with density-functional theory [44]. In the Kohn-Sham (KS) scheme, KS orbitals $\left[\varphi_{\sigma, j}(x),(\sigma=\downarrow, \uparrow)\right]$ are determined by

$$
\left\{-\frac{1}{2} \frac{\partial^{2}}{\partial x^{2}}+v_{\mathrm{KS}}\left[n_{\sigma}\right](x)\right\} \varphi_{\sigma, j}(x)=\varepsilon_{\sigma, j} \varphi_{\sigma, j}(x),
$$

with the static KS potential

$$
v_{\mathrm{KS}}\left[n_{\sigma}\right](x)=v_{\text {ion }}(x)+v_{H}[n](x)+v_{\mathrm{xc}}\left[n_{\sigma}\right](x) .
$$

The Hartree potential is given as

$$
v_{H}[n](x)=\int d x^{\prime} \frac{n\left(x^{\prime}\right)}{\sqrt{\left(x-x^{\prime}\right)^{2}+\epsilon}},
$$

and the exchange-correlation potential is treated in the local spin-density approximation

$$
v_{\mathrm{xc}}\left[n_{\sigma}\right](x) \simeq v_{x}\left[n_{\sigma}\right](x)=-\left[6 n_{\sigma}(x) / \pi\right]^{1 / 3} .
$$

The spin densities for spin $\sigma=\downarrow, \uparrow$ and the total density reads

$$
n_{\sigma}(x)=\sum_{j=0}^{N_{\sigma}-1}\left|\varphi_{\sigma, j}(x)\right|^{2}, \quad n(x)=\sum_{\sigma=\downarrow, \uparrow} n_{\sigma}(x) .
$$

We use the local spin-density approximation for the threedimensional electron gas since we aim at modeling HHG from a three-dimensional system rather than solving a 1D system exactly. As shown in Ref. [30], this approach captures the main features of HHG in a bulk solid.

The interaction of our systems with the laser fields is simulated using TDDFT. In the adiabatic approximation [45], the stationary KS equation (3) is replaced by the time-dependent KS equation

$$
i \frac{\partial}{\partial t} \varphi_{\sigma, j}(x, t)=\left\{-\frac{1}{2} \frac{\partial^{2}}{\partial x^{2}}-i A(t) \frac{\partial}{\partial x}+\tilde{v}_{\mathrm{KS}}\left[n_{\sigma}\right](x, t)\right\} \varphi_{\sigma, j}(x, t),
$$


where the KS potential reads

$$
\tilde{v}_{\mathrm{KS}}\left[n_{\sigma}\right](x, t)=v_{\text {ion }}(x)+v_{H}[n](x, t)+v_{\mathrm{xc}}\left[n_{\sigma}\right](x, t) .
$$

In this study, the driving laser pulse is linearly polarized along the $x$ axis and the vector potential is given as

$$
A(t)=A_{0} \sin ^{2}\left[\omega_{0} t /\left(2 n_{\mathrm{cyc}}\right)\right] \sin \left(\omega_{0} t\right),
$$

for $0 \leqslant t \leqslant 2 \pi n_{\text {cyc }} / \omega_{0}$ and zero otherwise, where $\omega_{0}$ and $n_{\text {cyc }}$ are the angular frequency and the number of cycles of the laser pulse. Here $A_{0}$ is the amplitude of the vector potential related to the field strength $F_{0}$ by $A_{0}=F_{0} / \omega_{0}$.

The KS orbitals were propagated in time according to the time-dependent KS equation (8) using the Crank-Nicolson method (with a predictor-corrector step) [46]. The system starts at time $t=0$ from the ground state with spin densities $n_{\sigma}(x, 0)=n_{\sigma 0}(x)$ and total density $n(x, 0)=n_{0}(x)$. The dynamic KS potential is updated in each time step. For smallenough field strengths, there is almost no difference between the dynamic and static KS potentials $\left(\tilde{v}_{\mathrm{KS} 0}(x)=v_{\text {ion }}(x)+\right.$ $\left.v_{H}\left[n_{0}\right](x)+v_{\mathrm{xc}}\left[\left\{n_{\sigma 0}\right\}\right](x)\right)[30]$. The calculations that we have performed in connection with this study show that the main conclusions regarding effects of vacancies on HHG in the linear chain are unaffected whether a static or dynamic KS potential is used. For simplicity, we therefore focus on the results obtained using the static KS potential in the following.

The numerical calculations were performed on an equidistant spatial grid with spacing $\Delta x=0.1$ and 45000 grid points corresponding to a box ranging from $x \approx-321.5 a$ to $x \approx$ $321.5 a$. The extent of the physical system with 200 ions is from $x=-99.5 a$ to $x=99.5 a$. The initial conditions for the TDDFT calculations, i.e., the field-free ground-state KS orbitals, were found via imaginary time propagation with orthogonalization in each time step [47]. A fixed step size $\Delta t=0.5$ was used for imaginary time propagation. A fixed step size $\Delta t=0.1$ was used for real-time propagation and convergence was checked. The laser parameters will be given in Sec. III B.

\section{RESULTS AND DISCUSSION}

In this section, we discuss the influence of introducing vacancies on the field-free potentials and band structures within the KS scheme and how vacancies change the HHG spectrum compared to the vacancy-free system. In the rest of the paper, the indexes of the positions of the vacancies are showed in parentheses. For instance, 200(100) represents a system with length of $199 a$ in which the index of the vacancy is 100 . We denote a system with length of $199 a$ having vacancies at the site of every $m$ ion as 200-mul $(m)$, so, e.g., 200-mul(40) has vacancies at indexes of 40,80,120, and 160. From previous studies we know that a linear chain with more than 60-80 ions responds to the driving field like a bulk solid [32].

\section{A. Effects of vacancies on KS potentials and band structures}

Here we investigate how the field-free properties in the DFT description are changed by introducing vacancies in the system. First, we compare the systems with and without vacancies in terms of the static KS potential which is obtained by imaginary time propagation. The impact of inserting vacancies in a chain of ions is restricted in real space to a small

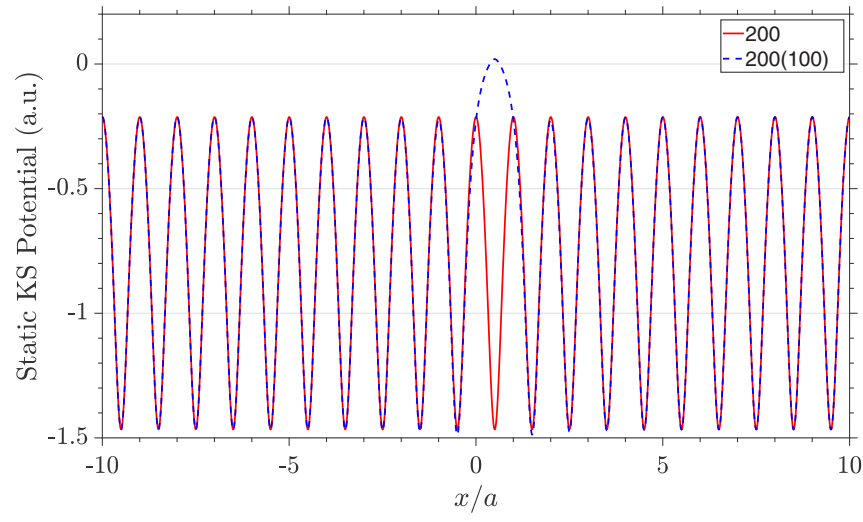

FIG. 1. Zoom-in of the central region of the static KS potential of 200 and 200(100) systems where the former is a perfect lattice with 200 ions and the latter has a single vacancy at the lattice position with index 100. Each local minimum of the KS potential corresponds to the position of a nucleus. The vacancy in the 200(100) system introduces a maximum (potential barrier).

region around its position. As an example, we consider in Fig. 1 the static KS potential of the single-vacancy 200(100) system compared with the system with no vacancy. This figure shows that the vacancy introduces a potential barrier around its position compared to the potential heights at neighboring positions. As we shall see later, this barrier to some extent leads to a partitioning of the lattice into subsystems. The motion of the electrons, however, is not entirely restricted by the barrier: When the electrons gain sufficient energy, they can pass the potential above the barrier.

From the static KS potential, we obtain the occupied and unoccupied KS orbitals and their corresponding energies through diagonalization. It should be noticed that in the KS scheme, the classification into occupied and unoccupied orbitals is automatically determined by the number of electrons and the Pauli exclusion principle.

We can classify the orbital energies into bands, since our model behaves like a solid for the considered large system sizes [32]. The band structures can be constructed from the Fourier-transformed orbitals (in $k$ space), as done, e.g., in Refs. [30,32]. Figure 2 shows the norm square of the $k$-space KS orbitals for illustrative examples of systems with and without vacancies. The energy range in this plot includes the highest valence band (VB) and two conduction bands (CB1 and $\mathrm{CB} 2$ ). Since our simulations are performed in a finite box, the free-space (FS) dispersion $k^{2} / 2$ is also present in Fig. 2. For HHG in solids, however, the FS parabola does not play any noticeable role [30,32]. Finally, in this figure, the dashed (red) lines show the energies of the (b) two and (c) four defect-state orbitals introduced by the vacancies. According to Fig. 2, the band structures of systems with one and two vacancies are similar to the band structure of the system without any vacancy. However, as can be seen in Fig. 2(c), systems with evenly distributed vacancies display more structures in addition to the main band structures. This change indicates that the KS orbitals in these systems are less localized in $k$ space than those for the system without any vacancy. This difference in $k$-space localization can be understood in the real space picture. By introducing 

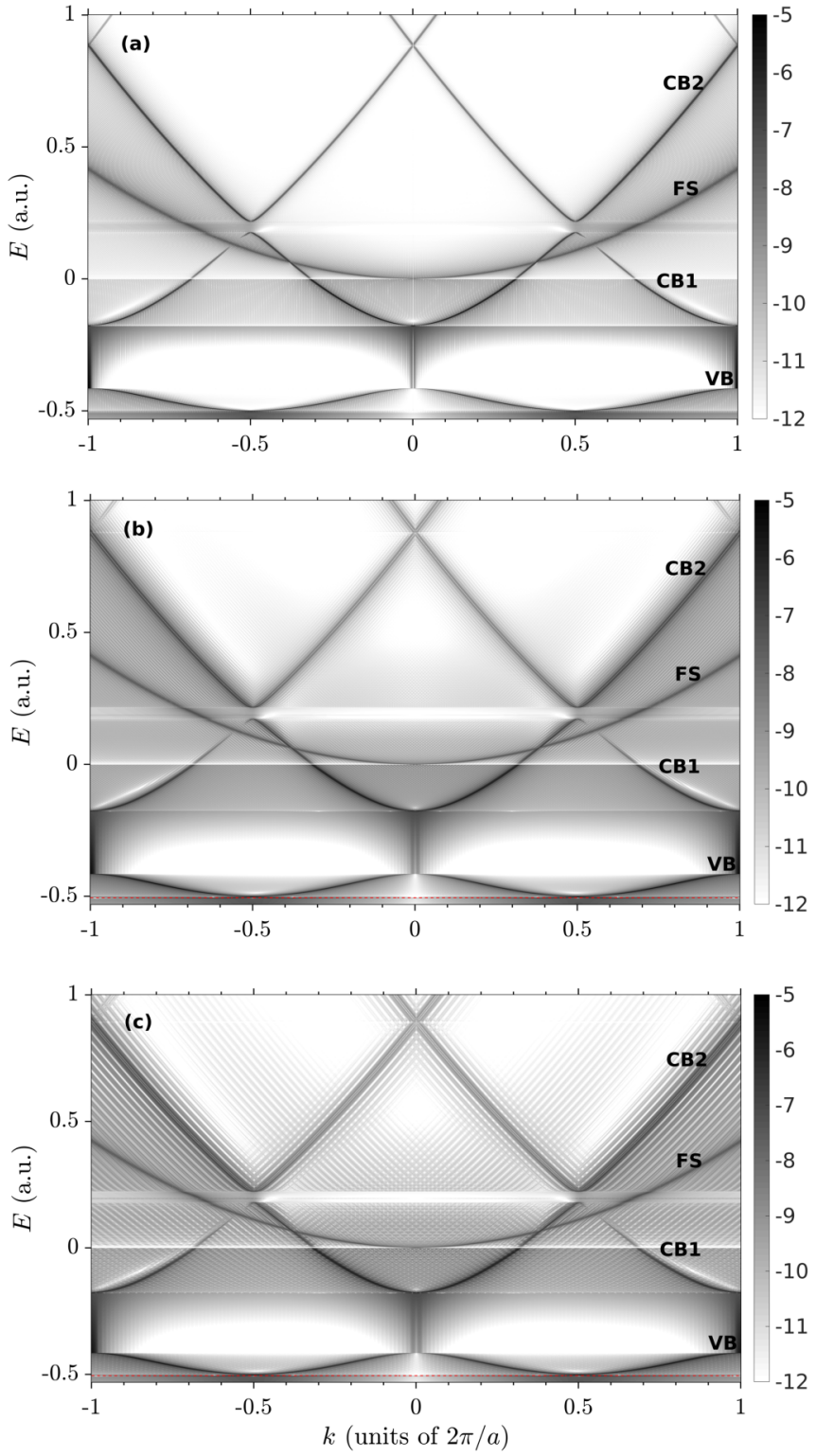

FIG. 2. Band structures of (a) 200, (b) 200(100), and (c) 200mul(67) systems calculated by the norm square of the Fouriertransformed KS orbitals. The systems correspond to a perfect lattice with 200 ions, a lattice with a vacancy in the middle of the lattice at the lattice position with index 100 , and a lattice with vacancies at the lattice positions with indexes 67 and 134, respectively. The free-space (FS) dispersion $k^{2} / 2$ is visible due to the finiteness of the simulation box (see text). The first-Brillouin-zone boundary is at $k= \pm \pi / a \approx \pm 0.449$. In the figure, we show the energy range of the highest valence band labeled by VB and the two first conduction bands labeled by CB1 and CB2, respectively. The horizontal (red) dashed lines in panels (b) and (c) show the energy levels of defectstate orbitals. In the present system, two defect-state orbitals are introduced per defect, so in (b) there are two and in (c) there are four defect-state orbitals. Their energy differences cannot be resolved at the scale of the figure.

vacancies in the system, the $\mathrm{KS}$ orbitals mostly localize between vacancies due to the additional potential barriers (Fig. 1). We have performed calculations that show that if a limited number of vacancies are confined to a small localized region of the chain [e.g., 200(98)(99)(100)(101)(102) or in a compact notation 200(98to102)], then the band structure is similar to that of the system with just one vacancy. For the considered set of parameters the band-gap energies for systems with and without vacancies are equal up to the third digit. Finally, we note that for the present system, each vacancy leads to the introduction of two defect-state orbitals that are spatially localized around each vacancy. Accordingly, in Fig. 2(b) there are two defect-state orbitals and in Fig. 2(c) there are four defect-state orbitals. The energies of these latter orbitals cannot be distinguished on the scale of the figure. We will come back to the contribution to the HHG spectra from these defect-state orbitals in the next section.

\section{B. Effects of vacancies on the HHG spectra}

We use the ground-state occupied KS orbitals as the initial state and perform calculations with a static KS potential for the systems interacting with a 15-cycle laser pulse of angular frequency $\omega_{0}=0.023$ (corresponding to a wavelength of $1981 \mathrm{~nm}$ ) and $A_{0}=0.24$ (corresponding to an intensity of $\left.\sim 10^{12} \mathrm{~W} / \mathrm{cm}^{2}\right)$. The band gap between the highest $\mathrm{VB}$ and the first conduction band (CB1) at the $\Gamma$ point $(k=0)$ equals 0.235 a.u. $\sim 6.395 \mathrm{eV}$ which corresponds to approximately 10 times the carrier photon energy $0.626 \mathrm{eV}$. It means that at least 10 photons are needed to make a transition from the valence band to the conduction band. As a result, harmonics up to order 10 are in the sub-BG regime for the studied systems. This dynamic regime is similar to the one considered experimentally, where the minimum number of photons required for an excitation from the valence to the conduction band is typically between 10 and 20: A minimum number of 9 photons was required for $\mathrm{ZnO}$ [1], 13 for solid $\mathrm{Kr}$ [8], 15 for solid Ar [8], and 14 to 19 for bulk GaSe [5,6]. We note that the critical vector potential, estimated by equating the band-gap energy, $E_{\mathrm{bg}}$, with the energy in the field over a lattice spacing, $A_{\mathrm{cr}}=F_{\mathrm{cr}} / \omega_{0}=\left(E_{\mathrm{bg}} / a\right) / \omega_{0} \simeq 1.4$, is much larger than the considered vector potential.

The time-dependent current is calculated by adding the contributions to the current from the KS orbitals,

$$
J(t)=\sum_{\sigma, j} \int d x \operatorname{Re}\left\{\varphi_{\sigma, j}^{*}(x, t)\left[-i \frac{\partial}{\partial x}+A(t)\right] \varphi_{\sigma, j}(x, t)\right\},
$$

and the HHG spectral intensity is evaluated as the modulus square of the Fourier-transformed current, as given by

$$
S(\omega) \propto\left|\int d t J(t) \exp (-i \omega t)\right|^{2} .
$$

Hence, we do not account for macroscopic propagation effects, which may modify the HHG spectra via absorption and phase mismatch. Such effects, however, can be reduced by controlling the thickness of target materials [8]. Therefore, it is expected that our conclusions hold for a thin target material.

At first, we study the effect of introducing vacancies which are evenly distributed along the chain of ions. By introducing vacancies in the reference system, we divide it into several subsystems with smaller lengths. Figure 3 shows the HHG spectra for the reference system without any vacancy together with spectra obtained from systems with an increasing 


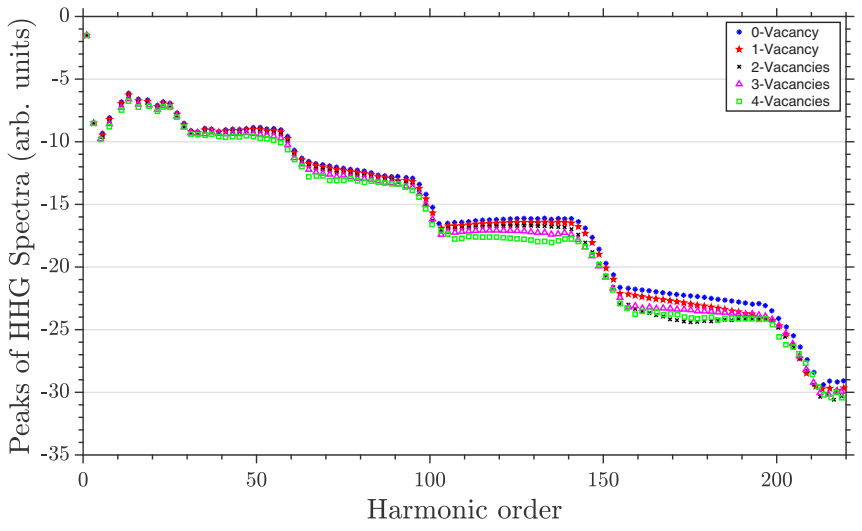

FIG. 3. Logarithm of the peaks of HHG spectra of systems with different number of vacancies evenly distributed in the chain of ions. The reference system consists of 200 ions with a lattice spacing of 7 a.u.. Adding more vacancies reduces the HHG peak signals until the fifth plateau, i.e., until harmonic order $\lesssim 140$. A 15-cycle laser pulse with wavelength of $1981 \mathrm{~nm}$ and intensity of $1.0665 \times 10^{12} \mathrm{~W} / \mathrm{cm}^{2}$ is used.

number of vacancies. All HHG spectra shown in Fig. 3 have similar overall structure with five plateaus and corresponding cutoffs. The cutoffs of these spectra can be determined by the maximum energy difference between the highest VB and the conduction bands (CB1, CB2, CB3, CB4, CB5) at a specific $k$ value being accessible from at least one initial $k_{0}$ (see Ref. [30] for more details about the origin of plateau cutoffs). At low order harmonics the maxima of the HHG spectra of these systems are relatively close to each other. At higher harmonics (especially after $\omega \simeq 100 \omega_{0}$ ) the maximum HHG peaks decrease in amplitude with increasing number of vacancies. At the 5th plateau with harmonic orders between 150 and 200, this trend still exist if we ignore the system with two vacancies. This exception appears in a regime with very small yield and it will therefore not be studied further in this work. To compare the harmonic yields of these systems quantitatively, we integrate the spectrum of the system with $m$ vacancies over harmonic orders 10 to 25 . The result of this integration is labeled by $I_{m}$. Figure 4 shows $I_{m} / I_{0}$ versus number of vacancies, and we see how the integrated spectrum in the first plateau decreases with increasing number of vacancies.

In our linear chain system, each vacancy introduces two defect-state orbitals that are spatially localized around the corresponding vacancy. These defect-state orbitals are occupied and their energies are located slightly below the lowest energy orbital in the valance band [see dashed (red) horizontal lines in Figs. 2(b) and 2(c)]. It is instructive to consider the role of these defect-state orbitals in the HHG process. To do so, we introduce the HHG-orbital index profile plot which shows the HHG yield as a function of the orbital index $j=$ $0, \ldots,\left(N_{\sigma}-1\right)$. The orbital index specifies the orbital and its energy and therefore also whether it is a defect-state orbital or a VB orbital. The HHG yield generated by the $j$ th orbital is obtained by $\left|\int d t J_{j}(t) \exp (-i \omega t)\right|^{2}$, where

$$
J_{j}(t)=\sum_{\sigma} \int d x \operatorname{Re}\left\{\varphi_{\sigma, j}^{*}(x, t)\left[-i \frac{\partial}{\partial x}+A(t)\right] \varphi_{\sigma, j}(x, t)\right\} .
$$

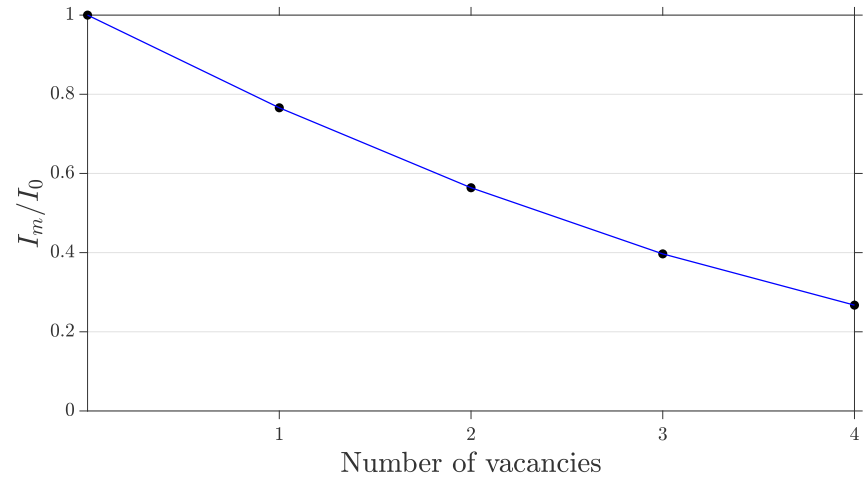

FIG. 4. Comparison of the HHG yield for harmonics in the first plateau of systems with different number of vacancies evenly distributed along the chain of ions. The ratio $I_{m} / I_{0}$ denotes the yield with $m$ vacancies relative to the yield with no vacancy and shows how the yield decreases with increasing $m$. The laser parameters are as in Fig. 3.

From the HHG-orbital index profile plot, it is easy to identify the contribution of the individual orbitals to the HHG signal. Figure 5 shows the HHG-orbital index profile of the system with four vacancies and for a 15-cycle pulse with $A_{0}=0.24$, $\lambda=1981 \mathrm{~nm}$ and using the static KS potential. In this figure, the eight orbital indexes from 199 to 206 correspond to

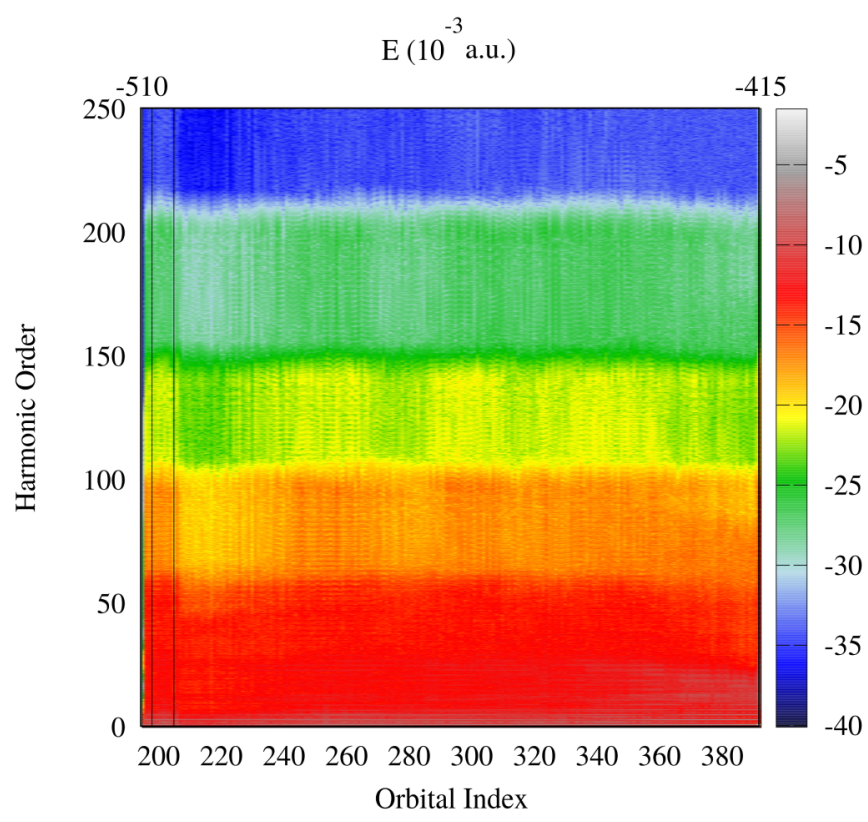

FIG. 5. HHG-orbital index profile plot of the system with four vacancies using the static KS potential. The vertical axis shows the harmonic orders from 0 to 250 . The color scale bar to the right in the figure shows the logarithm of the harmonic yield. The horizontal axis at the bottom of the figure shows the orbital indexes from 196 to 392. These indexes correspond to the defect-state and the VB orbitals. Each orbital has a particular energy. The energy range associated with the displayed orbital indexes is shown at the top of the figure in units of $10^{-3}$ a.u. (see also Fig. 2). The defect-state orbitals are located between the two vertical lines to the left in the figure and are seen to contribute significantly to the yield. The laser parameters are as in Fig. 3. 


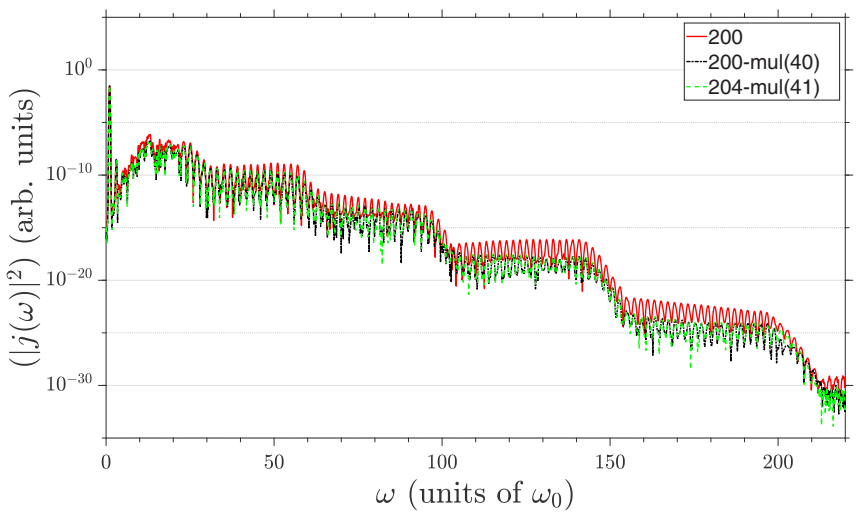

FIG. 6. Comparison of the HHG spectra of the 200 ion reference system, the system 200-mul(40) with four vacancies and the system 204-mul(41) with four vacancies. The similarity of the spectra of the two latter systems illustrate the insensitivity to the number of electrons in this parameter range. The laser parameters are as in Fig. 3.

defect-state orbitals and the orbital indexes from 207 to 392 belong to orbitals in the VB. The energy range of the orbitals with indexes from 197 to 392 is shown at the top of the figure (from -0.510 to -0.415 a.u.) and we see that this energy range is similar to the defect-state vs. VB energy range in Figs. 2(b) and 2(c). According to the results in Fig. 5, the defect-state orbitals, located in the figure between the two vertical black lines, contribute significantly to the HHG yield compared to, e.g., the VB orbitals with indexes around 220. We note that the importance of the defect states for the HHG process was recently reported in a study on hexagonal boron nitride [37].

Despite the importance of the defect-state orbitals in the HHG process in systems with vacancies [Fig. 5], we see from Figs. 3 and 4 that the introduction of vacancies leads to an overall decrease in the generation of harmonics. To identify why the systems with evenly distributed vacancies generate harmonics less efficiently than the reference system with no vacancy, we have made a number of considerations. First, we note that the changes in the band-gaps energies (in their third digit) with increasing number of vacancies are so small that they cannot explain the differences in the HHG yields based on Zener tunneling $\sim \exp \left[-a E_{\mathrm{bg}}^{2} /\left(4 \omega_{0} A_{0}\right)\right][48]$. Second, we considered possible effects related to the number of electrons involved in the HHG process, which is smaller in the system with vacancies than without. We therefore enlarged the systems with vacancies to keep the number of electrons fixed at the number in our 200 ion reference system. As an example, the number of electrons in the 204-mul(41) system with four evenly distributed vacancies and in the 200 system is identical, but their HHG spectra do not resemble each other (Fig. 6). However, the HHG spectra of the 204-mul(41) system is similar to the HHG spectra of the 200-mul(40) system, having a different number of electrons but identical number of vacancies and composed of subsystems with very similar length (see Fig. 6). We therefore conclude that the change in the HHG spectra in Fig. 3 is not directly associated with a change in the number of electrons participating in the generation process but rather a result of dividing the system into several subsystems by the change of potential, i.e., by the extra barriers effectively introduced in the KS potential by the vacancies (see Fig. 1). To this end, it is also useful to recall that the HHG spectrum can be expressed in terms of a Fourier transform (FT) of an expression involving the total density and the gradient of the ionic potential [29] as

$$
\mathrm{S}(\omega) \propto\left|\mathrm{FT}\left[\int d x n(x, t) \frac{\partial}{\partial x} v_{\text {ion }}(x)\right]\right|^{2} .
$$

Near the vacancies, the gradient of the ionic potential $\left[\frac{\partial}{\partial x} v_{\text {ion }}(x)\right]$ becomes smaller and the total electronic density $[n(x, t)]$ decreases drastically in such a way that the multiplication of these two terms, i.e., $n(x, t) \frac{\partial}{\partial x} v_{\text {ion }}(x)$, effectively reduces to zero. Consequently, by increasing the number of vacancies, no matter if they are localized or evenly distributed, the absolute value of the integral in Eq. (14) is decreased.

According to Eqs. (11) and (12), if we divide the reference system into $q$ subsystems, then $J_{\text {system }}=q J_{\text {subsystem }}$. Thus it can be expected that the HHG of a system which has several vacancies with similar distance between them [e.g., 200-mul $(m)$ ] can be modeled via the HHG of its subsystems by

$$
S(\omega)_{\text {system }} \simeq q^{2} S(\omega)_{\text {subsystem }},
$$

where $q$ represents the number of subsystems. If the subsystems are large enough to show bulklike behavior, i.e., if the number of ions $\gtrsim 80$ [32], then the above formula works reasonably accurately for the whole spectrum [Fig. 7(a)]. For smaller subsystems, the resulting total spectrum only partly resembles the main system's spectrum [only the lower harmonics are well described by Eq. (15), see Fig. 7(b)]. This again links the reduction in the yield (Figs. 3 and 4) to a finite-size effect related to a division into subsystems by the effective KS potential (Fig. 1).

As discussed in Ref. [32], for system sizes between the atomic system and the bulklike limit, the finite size restricts the movement of the electron-hole pair, limiting its propagation length to be smaller than the system size which leads to a linear dependence of the HHG cutoff on the system size. So there are smaller cutoffs for smaller systems and we expect to see the imprinting signature of the finite-size subsystems in the main HHG spectrum. According to Fig. 7(b), at low harmonics the HHG spectrum of the 200-mul(20) system composed of subsystems with finite structure (20), resembles the HHG spectrum of its subsystems as described by Eq. (15), but it is different from the HHG spectrum of the reference system (200). On the other hand, the number of states in the valence band of the 200-mul(20) system enables a harmonic generation process similar to the 200 system. As a result, the 200-mul(20) HHG spectrum has several plateaus similar to the 200 HHG spectrum. The reason that this system with vacancies still produces such a spectrum is that some electrons gain enough energy to overcome the effective barriers introduced by the vacancies (see Fig. 1), and these electrons contribute to the generation of harmonics from the bulk.

Interestingly, if we localize a limited number of vacancies next to each other to mimic a molecular-type point defect, then the overall HHG spectrum is similar to that of the system with just a single vacancy. As shown in Fig. 8(a), the HHG 

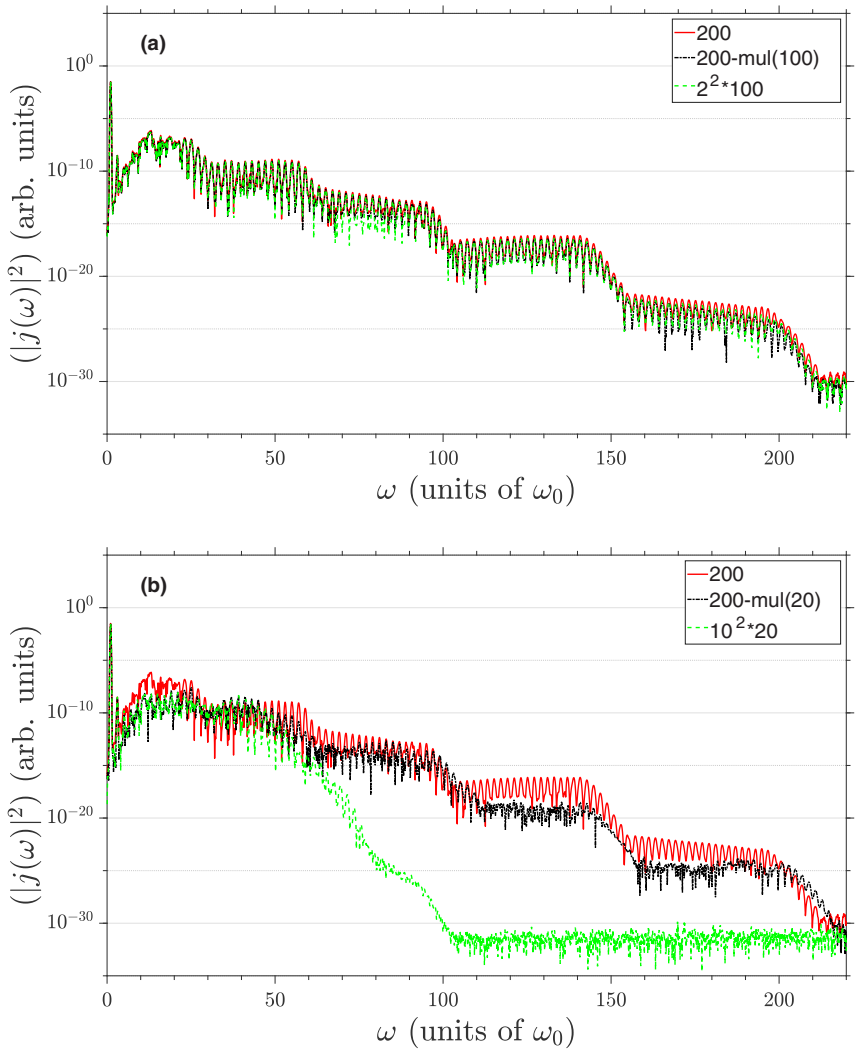

FIG. 7. (a) Comparison of the HHG spectrum of the 200 ion reference system with the spectrum of the 200-mul(100) system with a single vacancy and the spectrum obtained from Eq. (15) and a system with 100 ions. (b) HHG spectra of the 200-ion reference system, the 200-mul(20) system, and the spectrum obtained from the 20-ion system and Eq. (15). The laser parameters are as in Fig. 3.

spectra of 200(100) (a system with only a single vacancy) and 200(98to102) (a system with five vacancies localized next to each other) are very similar because of the similarity of the KS potential of these two systems. Both of them have a barrier in the middle and two almost similar subsystems, see Fig. 8(b).

We also used a time-frequency profile analysis to see how the HHG spectra of the systems with and without vacancies are built up in the time domain. In order to obtain these profiles, we perform a Gabor transform of the current $J(t)$ given in Eq. (11) by calculating

$$
G(\omega, t)=\int d t^{\prime} J\left(t^{\prime}\right) \exp \left(-i \omega t^{\prime}\right) \exp \left[-\frac{\left(t-t^{\prime}\right)^{2}}{2 \tau^{2}}\right]
$$

where the width of the time window $\tau$ is chosen to be $5 \pi$ (a.u.). The Gabor spectra $|G(\omega, t)|^{2}$ for the systems with and without vacancies are shown in Fig. 9. Figure 9(a) shows the result for the reference system. According to Fig. 9(b), the system with four evenly distributed vacancies shows fewer regular sinusoidal structures in the third plateau $(\omega=65-$ $\left.100 \omega_{0}\right)$, especially after a time of eight laser cycles. This feature is induced by the new periodicity added to the lattice periodicity by introducing vacancies in the chain. For the system with nine vacancies [Fig. 9(c)], the blurring out of the sinusoidal pattern is more significant at high harmonic orders.
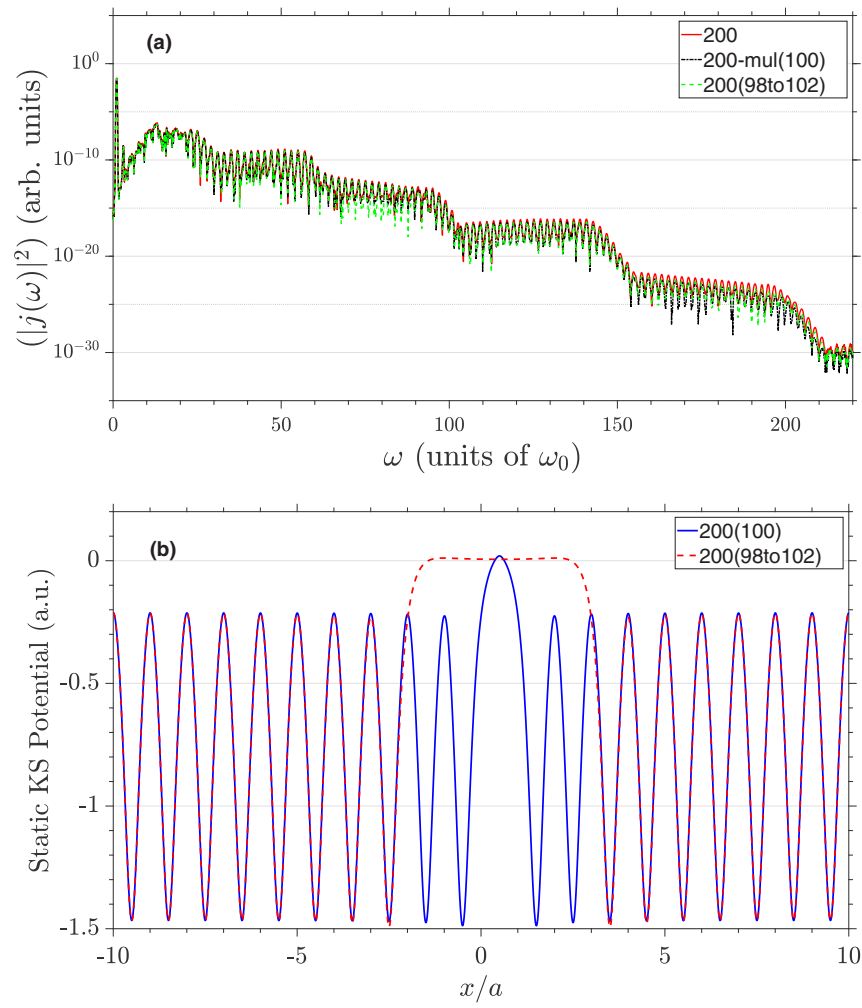

FIG. 8. (a) HHG spectra of the 200-ion reference system, the 200(100) system with a single vacancy in the middle of the linear chain, and the 200(98to102) system with five vacancies localized next to each other in the middle of the linear chain. (b) Zoom-in of the central region of the static KS potential of systems with one and five localized vacancies [200(100) and 200(98to102) systems]. The laser parameters are as in Fig. 3.

As shown in Ref. [35], in the case of disorder in the ionic positions in the lattice, the emission of harmonics is also less regular in time. However, in this case, the irregularity is induced by breaking the lattice periodicity by random shifts of ionic locations. Very recently, the appearance of ill-resolved electron trajectories in the Gabor profile of systems with vacancies were related to the interference of additional paths for the electron dynamics introduced by the defect states [49].

Once a sufficiently large number of ions $(\gtrsim 80)$ is considered, the results are insensitive to the system size. Our results regarding the effects of vacancies are also insensitive to the location of the atomic-type point vacancies when the number of vacancies in the two systems are exactly the same (for instance, we have checked that the HHG spectra of 200(10) and 200(100) are very similar to each other). Thus the results are independent of the symmetry of systems. Furthermore, we have performed additional calculations to confirm the reported trends in our results for a range of laser intensities from $8.6384 \times 10^{11}$ to $1.2904 \times 10^{12} \mathrm{~W} / \mathrm{cm}^{2}$.

Finally, we study a second realization of our linear chain model to be able to obtain a more complete picture of the possible effects of vacancy defects in HHG. As in Ref. [35], we set $N=200, \epsilon=2, a=4$, and $Z_{i}=2[i=0, \ldots,(N-1)]$ in Eq. (2) and use an eight-cycle laser pulse with a wavelength of $3036 \mathrm{~nm}$ and an intensity of $7.0875 \times 10^{11} \mathrm{~W} / \mathrm{cm}^{2}$. An 

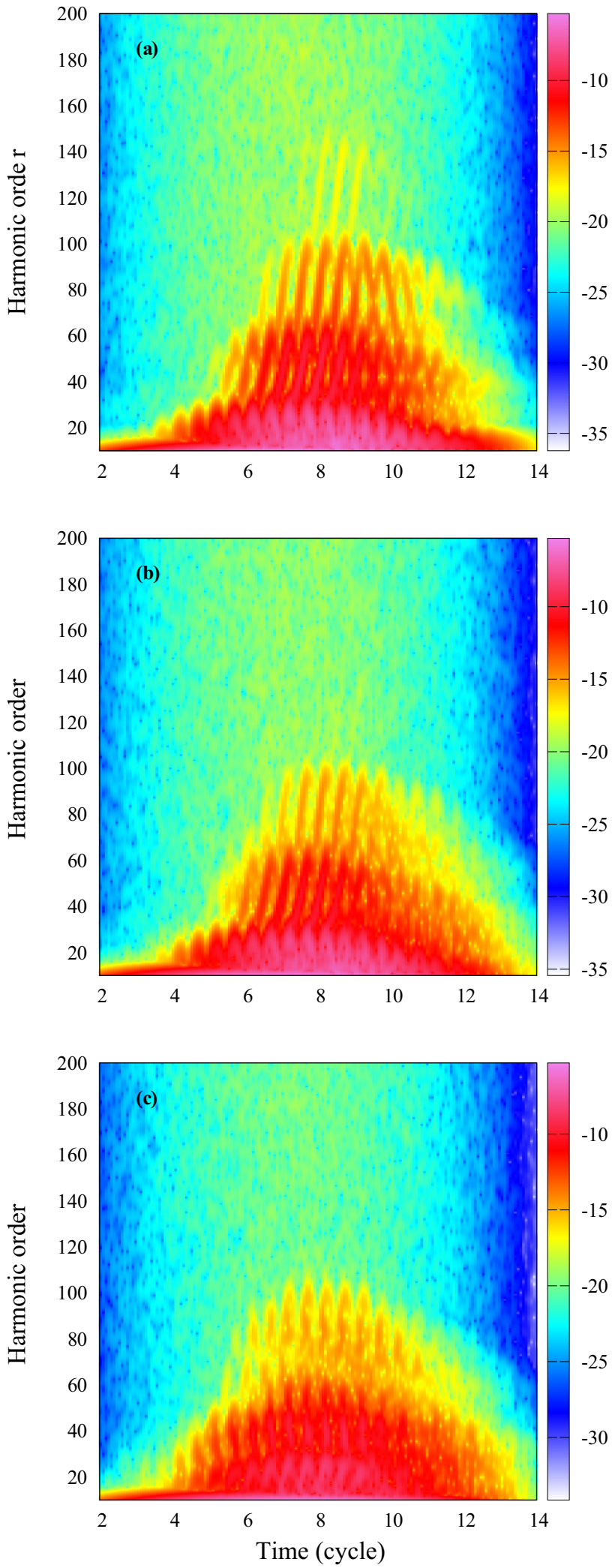

FIG. 9. Time-frequency profile of (a) the 200 ion reference system, (b) the 200-mul(40) system with four evenly distributed vacancies, and (c) the 200-mul(20) system with nine evenly distributed vacancies. The color scale bars to the right in the figure show the logarithm of the harmonic yield. The laser parameters are as in Fig. 3.

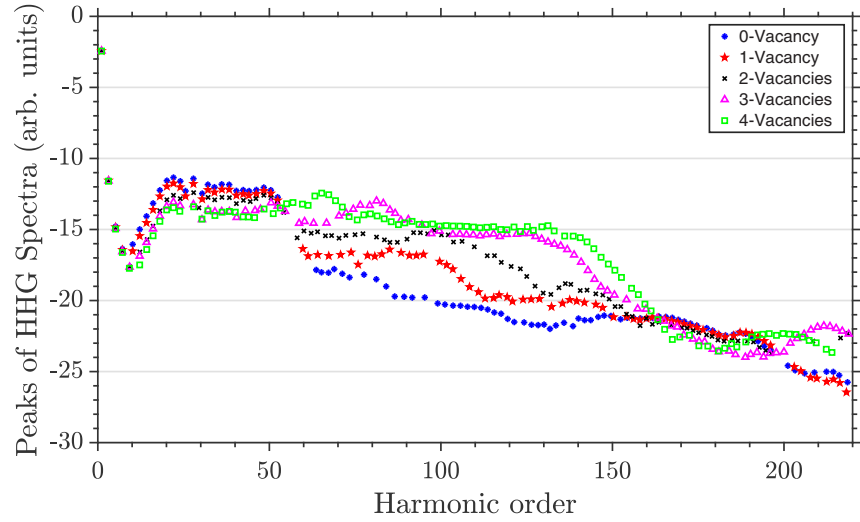

FIG. 10. Logarithm of the peaks of HHG spectra of a spinand charge-neutral linear chain with $Z_{i}=2$ and a lattice spacing of $a=4$. The figure shows the peaks of the HHG spectra for different number of vacancies evenly distributed in the chain. Adding more vacancies reduces the HHG peak signals until the second plateau, i.e., until harmonic order $\lesssim 50$. Interestingly, the harmonic yield increases in the second plateau by increasing the number of vacancies. An 8-cycle laser pulse with wavelength of $3036 \mathrm{~nm}$ and intensity of $7.0875 \times 10^{11} \mathrm{~W} / \mathrm{cm}^{2}$ is used.

illustration of the band structure can be found in Ref. [35]. In this system each vacancy introduces two defect-state orbitals. Their energy position is at the bottom of the VB. Figure 10 shows the HHG spectra for this chain without any vacancy together with spectra obtained from systems with an increasing number of vacancies. All HHG spectra shown in this figure have similar overall structure with two plateaus. By introducing vacancies in this second chain, the harmonic yield decreases in the first plateau and interestingly increases in the second plateau. Figure 11 shows $I_{m} / I_{0}$ versus number of vacancies and is obtained by integrating the spectrum of the system with $m$ vacancies (labled as $I_{m}$ ) over harmonic orders 20 to 50 (the first plateau). Comparing with Fig. 4, the harmonic yield of the first plateau of the spectrum decreases more rapidly with increasing number of vacancies. We have made

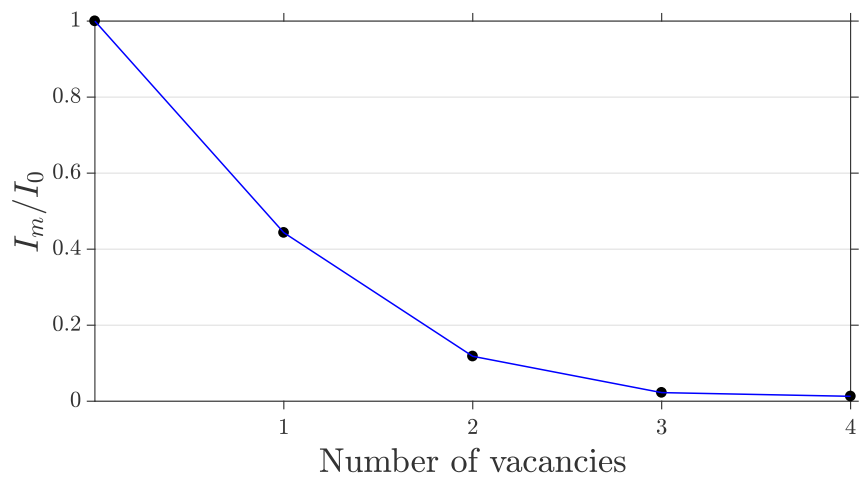

FIG. 11. Comparison of the HHG yields for the harmonics in the first plateau for the linear chain considered in Fig. 10 for different number of vacancies evenly distributed along the chain of ions. The ratio $I_{m} / I_{0}$ denotes the yield with $m$ vacancies relative to the yield with no vacancy and shows how the yield decreases with increasing $m$. The laser parameters are as in Fig. 10. 
HHG-orbital index profile plots also for the cases considered for this chain system but do not include these plots here. The conclusion from these profile plots is that there is no particular role of the defect-state orbitals in the HHG process for this chain, in opposition to the conclusions drawn from Fig. 5 for the other considered chain system, and illustrating that the role of defect-state orbitals can be very system dependent. As it is shown in Figs. 10 and 11, the shape of the HHG spectrum and the harmonic yield may change considerably by introducing vacancies in a linear chain system. A comparison with the results in Figs. 3 and 4, where we observed relatively small changes, show that the magnitude of such changes can be very sensitive to the chain system.

\section{CONCLUSION}

We employed a self-consistent time-dependent densityfunctional approach to study how the presence of vacancies in a perfect lattice changes the band structure and, consequently, the HHG spectrum of the lattice. We used a 1D model system of a linear chain of identical ions to produce a typical band structure and to capture the essential aspects of HHG in solids. We found that by introducing a limited number of evenly distributed vacancies, the band structure is almost not affected. In the case where several vacancies are confined to a small region of the chain, the band structure is similar to that of the system with just one vacancy. Because of the localization of the KS orbitals between vacancies (or between a vacancy and an edge of the chain), the systems with vacancies display more structures in their band structures. In the HHG spectra of the vacancy-free reference system and also for the systems with one to four vacancies, five plateaus appeared. The corresponding cutoffs can be determined by the maximum energy difference between the highest valence band and the first five conduction bands at a specific $k$ value. At low-order harmonics, the maximum peaks of the HHG spectra of these systems are relatively close to each other. At higher harmonics, the maximum HHG peaks decrease with increasing number of vacancies. We identified this change in the overall shape of the spectrum as originating from the additional effective potential barriers introduced at the positions of the vacancies, which means that only electrons having gained enough energy to pass above these barrier can effectively participate in HHG from the bulk. For one of the chain systems considered, we found that the induced defect-state orbitals are located energetically slightly below the lowest energy orbital in the VB. We found that the contribution from these former orbitals to the HHG spectrum may be more important than that from orbitals in the VB.
We showed that if the number of vacancies is limited, then the associated reduction in the number of electrons involved in the HHG process does not significantly affect the harmonic yield. We found that the number of evenly distributed vacancies (no matter if they are atomic- or molecular-type point vacancies) and the size of the subsystems are the factors which control the HHG yield. By introducing atomic-type point vacancies, the linear chain lattice is divided into several subsystems. We found that the HHG spectrum of a system with several vacancies can be modeled via the HHG of its subsystems if each of them is large enough to behave individually like a bulk solid, i.e., if it contains more that around 60-80 ions. Signatures of finite structure were detected in the HHG spectrum of a system composed of smaller subsystems, i.e., with smaller distance between the vacancies. Also in this case, the spectrum is composed of several plateaus resembling the overall structure of a bulk solid spectrum. Our results regarding overall changes in the spectra with vacancies are insensitive to the location of the atomic-type point vacancies and as a result to the symmetry of the system. Nevertheless, the shape of the spectra and the change with the number of vacancies may be very sensitive to the specific system. We illustrated this point by showing the significant difference in the change in the spectra with vacancies for two different realizations of the linear chain systems.

In our approach we ignored lattice relaxations around a vacancy and also the unpaired dangling bonds states at the vacancy site [50]. By considering these effects, more realistic imprinting of defects in the HHG spectra can be accessible [37]. Here we considered only spin and charge neutral vacancies; however, there is an interesting physics underlying F centers (anionic vacancies) [50]. With the advent of modern thinfilm technology, targeted manipulation and control of defects, in particular at their two-dimensional surfaces and interfaces, has become possible [51]. Moreover, theoretical investigation of defects is critical to understanding the electronic properties of semiconducting compounds [52]. As discussed here, the HHG spectra are sensitive to defects and vacancies, and in the future the time-resolved perspective associated with HHG (see, e.g., Ref. [53]) may be useful to shed new light on ultrafast dynamics in such systems.

\section{ACKNOWLEDGMENTS}

We thank Dieter Bauer for making the QPROP code, on which the TDDFT calculations are based, available. This work was supported by the Ministry of Science Research and Technology of Iran and the Villum-Kann Rasmussen center of excellence QUSCOPE-Quantum Scale Optical Processes. The numerical results presented in this work were obtained at the Centre for Scientific Computing, Aarhus.
[1] S. Ghimire, A. D. DiChiara, E. Sistrunk, P. Agostini, L. F. DiMauro, and D. A. Reis, Observation of high-order harmonic generation in a bulk crystal, Nat. Phys. 7, 138 (2011).

[2] S. Ghimire, A. D. DiChiara, E. Sistrunk, G. Ndabashimiye, U. B. Szafruga, A. Mohammad, P. Agostini, L. F. DiMauro, and D. A. Reis, Generation and propagation of high-order harmonics in crystals, Phys. Rev. A 85, 043836 (2012).
[3] M. Schultze, E. M. Bothschafter, A. Sommer, S. Holzner, W. Schweinberger, M. Fiess, M. Hofstetter, R. Kienberger, V. Apalkov, V. S. Yakovlev, M. I. Stockman, and F. Krausz, Controlling dielectrics with the electric field of light, Nature 493, 75 (2013).

[4] S. Ghimire, G. Ndabashimiye, A. D. DiChiara, E. Sistrunk, M. I. Stockman, P. Agostini, L. F. DiMauro, and D. A. Reis, 
Strong-field and attosecond physics in solids, J. Phys. B: At., Mol. Opt. Phys. 47, 204030 (2014).

[5] O. Schubert, M. Hohenleutner, F. Langer, B. Urbanek, C. Lange, U. Huttner, D. Golde, T. Meier, M. Kira, S. W. Koch, and R. Huber, Sub-cycle control of terahertz high-harmonic generation by dynamical bloch oscillations, Nat. Photon. 8, 119 (2014).

[6] M. Hohenleutner, F. Langer, O. Schubert, M. Knorr, U. Huttner, S. W. Koch, M. Kira, and R. Huber, Real-time observation of interfering crystal electrons in high-harmonic generation, Nature 523, 572 (2015).

[7] G. Vampa, T. J. Hammond, N. Thiré, B. E. Schmidt, F. Légaré, C. R. McDonald, T. Brabec, D. D. Klug, and P. B. Corkum, AllOptical Reconstruction of Crystal Band Structure, Phys. Rev. Lett. 115, 193603 (2015).

[8] G. Ndabashimiye, S. Ghimire, M. Wu, D. A. Browne, K. J. Schafer, M. B. Gaarde, and D. A. Reis, Solid-state harmonics beyond the atomic limit, Nature 534, 520 (2016).

[9] S. Yu. Kruchinin, F. Krausz, and V. S. Yakovlev, Colloquium: Strong-field phenomena in periodic systems, Rev. Mod. Phys. 90, 021002 (2018).

[10] G. Vampa and T. Brabec, Merge of high harmonic generation from gases and solids and its implications for attosecond science, J. Phys. B: At., Mol. Opt. Phys. 50, 083001 (2017).

[11] P. B. Corkum, Plasma Perspective on Strong Field Multiphoton Ionization, Phys. Rev. Lett. 71, 1994 (1993).

[12] J. L. Krause, K. J. Schafer, and K. C. Kulander, High-Order Harmonic Generation from Atoms and Ions in the High Intensity Regime, Phys. Rev. Lett. 68, 3535 (1992).

[13] G. Vampa, C. R. McDonald, G. Orlando, D. D. Klug, P. B. Corkum, and T. Brabec, Theoretical Analysis of HighHarmonic Generation in Solids, Phys. Rev. Lett. 113, 073901 (2014).

[14] R. Numico, D. Giulietti, A. Giulietti, L. A. Gizzi, and L. Roso, High-order harmonic generation from a linear chain of ions, J. Phys. B: At., Mol. Opt. Phys. 33, 2605 (2000).

[15] S. A. Sato and K. Yabana, Efficient basis expansion for describing linear and nonlinear electron dynamics in crystalline solids, Phys. Rev. B 89, 224305 (2014).

[16] M. Wu, S. Ghimire, D. A. Reis, K. J. Schafer, and M. B. Gaarde, High-harmonic generation from Bloch electrons in solids, Phys. Rev. A 91, 043839 (2015).

[17] P. G. Hawkins, M. Yu. Ivanov, and V. S. Yakovlev, Effect of multiple conduction bands on high-harmonic emission from dielectrics, Phys. Rev. A 91, 013405 (2015).

[18] M. Wu, D. A. Browne, K. J. Schafer, and M. B. Gaarde, Multilevel perspective on high-order harmonic generation in solids, Phys. Rev. A 94, 063403 (2016).

[19] C. Yu, X. Zhang, S. Jiang, X. Cao, G. Yuan, T. Wu, L. Bai, and R. Lu, Dependence of high-order-harmonic generation on dipole moment in $\mathrm{SiO}_{2}$ crystals, Phys. Rev. A 94, 013846 (2016).

[20] T. Ikemachi, Y. Shinohara, T. Sato, J. Yumoto, M. KuwataGonokami, and K. L. Ishikawa, Trajectory analysis of highorder-harmonic generation from periodic crystals, Phys. Rev. A 95, 043416 (2017).

[21] X. Liu, X. Zhu, P. Lan, X. Zhang, D. Wang, Q. Zhang, and P. Lu, Time-dependent population imaging for high-order-harmonic generation in solids, Phys. Rev. A 95, 063419 (2017).
[22] G. Vampa, C. R. McDonald, G. Orlando, P. B. Corkum, and T. Brabec, Semiclassical analysis of high harmonic generation in bulk crystals, Phys. Rev. B 91, 064302 (2015).

[23] M. S. Wismer, S. Yu. Kruchinin, M. Ciappina, M. I. Stockman, and V. S. Yakovlev, Strong-Field Resonant Dynamics in Semiconductors, Phys. Rev. Lett. 116, 197401 (2016).

[24] C. R. McDonald, K. S. Amin, S. Aalmalki, and T. Brabec, Enhancing High Harmonic Output in Solids Through Quantum Confinement, Phys. Rev. Lett. 119, 183902 (2017).

[25] U. Huttner, M. Kira, and S. W. Koch, Ultrahigh off-resonant field effects in semiconductors, Laser Photon. Rev. 11, 1700049 (2017).

[26] T. Otobe, K. Yabana, and J.-I. Iwata, First-principles calculation of the electron dynamics in crystalline $\mathrm{SiO}_{2}$, J. Phys.: Condens. Matter 21, 064224 (2009).

[27] T. Otobe, High-harmonic generation in $\alpha$-quartz by electronhole recombination, Phys. Rev. B 94, 235152 (2016).

[28] N. Tancogne-Dejean, O. D. Mücke, F. X. Kärtner, and A. Rubio, Ellipticity dependence of high-harmonic generation in solids originating from coupled intraband and interband dynamics, Nat. Commun. 8, 745 (2017).

[29] N. Tancogne-Dejean, O. D. Mücke, F. X. Kärtner, and A. Rubio, Impact of the Electronic Band Structure in High-Harmonic Generation Spectra of Solids, Phys. Rev. Lett. 118, 087403 (2017).

[30] K. K. Hansen, T. Deffge, and D. Bauer, High-order harmonic generation in solid slabs beyond the single-active-electron approximation, Phys. Rev. A 96, 053418 (2017).

[31] I. Floss, C. Lemell, G. Wachter, V. Smejkal, S. A. Sato, X.-M. Tong, K. Yabana, and J. Burgdörfer, Ab initio multiscale simulation of high-order harmonic generation in solids, Phys. Rev. A 97, 011401(R) (2018).

[32] K. K. Hansen, D. Bauer, and L. B. Madsen, Finite-system effects on high-order harmonic generation: From atoms to solids, Phys. Rev. A 97, 043424 (2018).

[33] D. Bauer and K. K. Hansen, High-Harmonic Generation in Solids with and without Topological Edge States, Phys. Rev. Lett. 120, 177401 (2018).

[34] C. Yu, K. K. Hansen, and L. B. Madsen, Enhanced high-order harmonic generation in donor-doped band-gap materials, Phys. Rev. A 99, 013435 (2019).

[35] C. Yu, K. K. Hansen, and L. B. Madsen, High-order harmonic generation in imperfect crystals, Phys. Rev. A 99, 063408 (2019).

[36] H. Drüeke and D. Bauer, Robustness of topologically sensitive harmonic generation in laser-driven linear chains, Phys. Rev. A 99, 053402 (2019).

[37] Mrudul M. S., N. Tancogne-Dejean, A. Rubio, and G. Dixit, High-Harmonic generation from spin-polarised defects in solids, npj Comput. Mater. 6, 10 (2020).

[38] T. Ikemachi, Y. Shinohara, T. Sato, J. Yumoto, M. KuwataGonokami, and K. L. Ishikawa, Time-dependent Hartree-Fock study of electron-hole interaction effects on high-order harmonic generation from periodic crystals, Phys. Rev. A 98, 023415 (2018).

[39] C. Jürß and D. Bauer, High-harmonic generation in Su-Schrieffer-Heeger chains, Phys. Rev. B 99, 195428 (2019). 
[40] C. Freysoldt, B. Grabowski, T. Hickel, J. Neugebauer, G. Kresse, A. Janotti, and C. G. Van de Walle, First-principles calculations for point defects in solids, Rev. Mod. Phys. 86, 253 (2014).

[41] R. A. Evarestov, Quantum Chemistry of Solids: The LCAO First Principles Treatment of Crystals (Springer, Berlin, 2007).

[42] R. A. Evarestov, Trends in calculation of point and extended defects in wide-gap solids: Periodic models of aperiodic systems, Phys. Status Solidi A 202, 235 (2005).

[43] J. Bernholc, N. O. Lipari, and S. T. Pantelides, Scatteringtheoretic method for defects in semiconductors. II. Selfconsistent formulation and application to the vacancy in silicon, Phys. Rev. B 21, 3545 (1980).

[44] R. M. Dreizler and E. K. U. Gross, Density Functional Theory: An Approach to the Quantum Many-Body Problem (Springer, Berlin, 1990).

[45] C. A. Ullrich, Time-dependent Density-Functional Theory: Concepts and Applications (Oxford University Press, Oxford, 2011).

[46] D. Bauer, Computational Strong-field Quantum Dynamics: Intense Light-matter Interactions, edited by Dieter Bauer (De Gruyter, Berlin, 2017), Chap. IV, pp. 131-132.

[47] D. Bauer and T. Fennel, Computational Strong-field Quantum Dynamics: Intense Light-matter Interactions, edited by Dieter Bauer (De Gruyter, Berlin, 2017), Chap. I, pp. 10-12.

[48] C. Zener, A theory of the electrical breakdown of solid dielectrics, Proc. R. Soc. Lond. A 145, 523 (1934).

[49] A. Pattanayak, Mrudul M. S., and G. Dixit, Influence of vacancy defects in solid high-order harmonic generation, Phys. Rev. A 101, 013404 (2020).

[50] J.-M. Spaeth and H. Overhof, Point Defects in Semiconductors and Insulators: Determination of Atomic and Electronic Structure from Paramagnetic Hyperfine Interactions (Springer, Berlin, 2003).

[51] M. Sing, H. O. Jeschke, F. Lechermann, R. Valentí, and R. Claessen, Influence of oxygen vacancies on two-dimensional electron systems at $\mathrm{SrTiO}_{3}$-based interfaces and surfaces, Eur. Phys. J.: Spec. Top. 226, 2457 (2017).

[52] L. L. Baranowski, P. Zawadzki, S. Lany, E. S. Toberer, and A. Zakutayev, A review of defects and disorder in multinary tetrahedrally bonded semiconductors, Semicond. Sci. Technol. 31, 123004 (2016).

[53] P. M. Kraus, B. Mignolet, D. Baykusheva, A. Rupenyan, L. Horný, E. F. Penka, G. Grassi, O. I. Tolstikhin, J. Schneider, F. Jensen, L. B. Madsen, A. D. Bandrauk, F. Remacle, and H. J. Wörner, Measurement and laser control of attosecond charge migration in ionized iodoacetylene, Science 350, 790 (2015). 